\title{
2 Objectives of this work
}

In this work, epithelial skin tumors (basal cell carcinoma and cutaneous squamous cell carcinoma) and malignant melanoma, as well as cutaneous melanoma metastases, are discussed.

In all three tumor entities, the expression of the most important components of the miRNA machinery (miRNA maturity, transport, and effect) are analyzed. Then, a characterization of differentially expressed miRNAs is made using the determination and analysis of miRNA expression profiles. The results of these studies should answer the following questions:

- $\quad$ Can significant differences in mRNA expression of the components of the miRNA machinery Dicer, Drosha, Argonaute-1, Argonaute-2, PACT, TARBP1, and TARBP2 be demonstrated using RT-PCR in basal cell carcinomas, cutaneous squamous cell carcinomas (cSCCs), and actinic keratoses (as pre-cancerous forms leading to cSCC)?

- $\quad$ Can significant differences of the Dicer expression be demonstrated at the protein level in malignant melanoma using immunohistochemical analysis?

- Can significant differences in mRNA expression of the components of the miRNA machinery Dicer, Drosha, Argonaute-1, Argonaute-2, PACT, TARBP1, TARBP2, MTDH, SND-1, and Exp-5 be demonstrated using RT-PCR in malignant melanoma and in cutaneous melanoma metastases?

- $\quad$ Can differentially expressed miRNAs be defined using microarraybased miRNA expression profiles in basal cell carcinomas, cutaneous squamous cell carcinomas, malignant melanomas, and cutaneous melanoma metastases? 